\title{
Reliability of Diffusion Weighted MRI for the Diagnosis of Residual and Recurrent Cholesteatoma

\author{
Medany Mahmoud Medany ${ }^{1}$, Reda Mohammed Sabra ${ }^{1}$, Ehab Kamal Hakim ${ }^{1}$, Amr Mohammed \\ Ismaeel Sadawy ${ }^{2}$, Ahmed Mohammed Medhat Elshafei ${ }^{1^{*}}$ \\ Departments ${ }^{1}$ Otorhinolaryngology and ${ }^{2}$ Radiodiagnosis, Faculty of Medicine, Ain Shams University \\ *Corresponding Author: Ahmed Mohammed Medhat Elshafei, E-Mail: Ahmedmedhats2006@gmail.com
}

\begin{abstract}
Background: olesteatoma is defined as the presence of collections of kertatinized squamous epithelium within a sac in temporal bone \& skull base, commonly involve the middle ear cavity \& mastoid cavities. Congenital cholesteatomas compose only $2 \%$ of middle ear cholesteatomas.

Aim of the Work: to evaluate the role of DWMRI in detecting residual and/or recurrent cholesteatoma and to compare it to the intra-operative findings.

Patients and Methods: this study was conducted on patients with recurrent or residual cholesteatoma suspected clinically or by CT examination at Ain Shams University hospitals or Hearing and Speech Institute. The patients were referred from the department of Otolaryngology, Ain Shams University hospitals, Hearing and Speech Institute and also from other private clinics.

Results: from the 30 cases we examined, 19 cases $(63 \%)$ showed areas of DWI restriction within the middle ear denoting primary or recurrent cholesteatoma. The smallest size of cholesteatoma detected was $4.5 \mathrm{~mm}$. All 19 cases that showed evidence of recurrent cholesteatoma on MS-EPI DWI MRI images underwent second look mastoid surgery will all cases (100\%) showing intra-operative evidence of cholesteatoma. Of the 11 cases that were negative for cholesteatoma on DWIs, 9 of these cases underwent second look mastoid surgery with 4 cases (44\%) showing small cholesteatomas measuring less than $4 \mathrm{~mm}$ while the other 5 cases (56\%).

Conclusion: DWI is a beneficial tool in the evaluation of recurrent cholesteatoma with excellent specificity that reduces the need of second look surgeries in multiple cases.
\end{abstract}

Keywords: Diffusion Weighted MRI, Residual, Recurrent Cholesteatoma

\section{INTRODUCTION}

Cholesteatoma is defined as the presence of collections of kertatinized squamous epithelium within a sac in temporal bone \& skull base, commonly involve the middle ear cavity \& mastoid cavities.It may be congenital or acquired. Congenital cholesteatomas compose only $2 \%$ of middle ear cholesteatomas (1). Cholesteatomas involve Prussak's space and progressively erodes the adjacent structures such as the scutum and the ossicular chain. After growth, the cholesteatoma invades the antrum and the mastoid process, eroding further structures of the middle ear such as the facial nerve canal, the tegmen tympani and the posterior semicircular canal wall ${ }^{(2)}$. It can erode and destroy important structures with potential for causing central nervous system (CNS) complications (eg, brain abscess, meningitis) makes it a potentially fatal lesion ${ }^{(3)}$. It is usually diagnosed on basis of history, otoscopic examination with radiological and audiological assessment. An understanding of the pathophysiology of aural cholesteatoma is important, due to it's destructive nature which is responsible for the morbidities associated with it's chronic nature. the lack of effective nonsurgical management add importance to the understanding of this disease, otomicroscopic evaluation is the principal diagnostic tool for acquired cholesteatoma, an inflammatory polyp or granulation tissue might be obscuring the vision on examination by, as recurrent or residual disease is mainly attributed to hidden areas that cannot be seen using surgical microscope. otoendoscopic examination and surgical exploration is other tools to be used for such cases ${ }^{(4)}$. A cholesteatoma can only be eradicated from the temporal bone by surgical resection and is usually managed with radical or modified radical mastoidectomy. The choice of surgical approach depends upon the scoring of the anatomical extentsion of the cholesteatoma into the middle ear and mastoid cavity and the status of the ossicular chain and tympanic membrane, selection of the optimal surgical approach results in complete disease eradication. these procedures can be classified into canal wall up and canal wall down procedures ${ }^{(5)}$. The incidence of recurrence of the chronic active disease vary from 3 to $18 \%$ of all surgical procedures and the failure most importantly attributed to residual or recurrent cholesteatoma with persistent suppuration of middle ear and mastoid ${ }^{(6)}$. Recurrent disease or disease due to denovo retraction (i.e. retraction pocket cholesteatoma) 
caused by scutum defects leading to recurrence of the primary pathology. Confirming a diagnosis recurrent or recurrent cholesteatoma through imaging techniques remains a challenge for the otolaryngologist \& head and neck radiologist. Imaging procedures such as high resolution computed tomography (CT) and magnetic resonance imaging (MRI) may suggest the presence of cholesteatomas within the temporal bone and may be used to complement the clinical examination. Selection of the appropriate modality of imaging depends on clinical findings as each of them provides different anatomical and pathological information undetectable by the other modality. Advanced technology, as multidetector row scanning with submillimeter $(0.5 \mathrm{~mm})$ section thickness and high rotation ( 0.5 second per rotation), has reinforced the value of CT scan for detecting temporal bone pathologies ${ }^{(7)}$. Unfortunately many studies have shown that HRCT is inaccurate in detecting post-operative residual and recurrent cholesteatoma, If used alone it can be misleading as it cannot differentiate between cholesteatoma, cholesterol granuloma, granulation, brain or fibrous tissue and mucoid secretion in the postmastoidectomy cavities and completely or partially opacified middle ears. This was the reason that clinicians sought alternative imaging modalities for better evaluation of the various types of tissues that may be present in ears that had undergone surgery (6). The MRI has an additional value in patients with prior cholesteatoma resection, particularly when CT findings are equivocal, is mainly due to its capacity to unequivocally confirm the diagnosis of cholesteatoma in cases of clinical doubt. It can distinguish cholesteatoma from other soft tissues, such as fibrosis, granulation tissue, inflammatory changes and cholesterol granuloma. It has the potential to document invasion of the labyrinth and of the intracranial space ${ }^{(8)}$. Recent studies on diffusion-weighted magnetic resonance imaging scans reported high rates of sensitivity $(85.2 \%-$ $90 \%$ ), specificity $(92.6 \%-100 \%)$, positive predictive value $(92.6 \%-100 \%)$ and negative predictive value (92\%) in the diagnosis of cholesteatomatous tissue in patients who had undergone tympanomastoid surgery ${ }^{(9)}$.

\section{AIM OF THE WORK}

To evaluate the role of DWMRI in detecting residual and/or recurrent cholesteatoma and to compare it to the intra-operative findings.

\section{PATIENTS AND METHODS}

This study was conducted on patients with recurrent or residual cholesteatoma suspected clinically or by CT examination at Ain Shams University hospitals or Hearing and Speech Institute. The patients were referred from the department of Otolaryngology, Ain Shams University hospitals, Hearing and Speech Institute and also from other private clinics. The patients were investigated using magnetic resonance imaging (MRI) preoperative, using the diffusion weighted protocols, to evaluate the extent of the cholesteatoma.. The study was approved by the Ethics Board of Ain Shams University and an informed written consent was taken from each participant in the study. Diffusion-weighted imaging is a technique that measures the molecular diffusion of water (Brownian motion) within the tissues being measured. Molecular diffusion refers to the random displacement of any type of molecule in a fluid (e.g., water), as the molecule is agitated by thermal energy. This erratic motion is best described in statistical terms by a displacement distribution. The displacement distribution describes the proportion of molecules that undergo displacement in a specific direction and to a specific distance. This can be helpful for diagnosis of cholesteatoma, since the high content of keratin of cholesteatoma is associated with restricted diffusion. Study place: Ain Shams University Hospitals, Cairo, Egypt. Sample size: 30 patients. Equipment used: For the MRI examination, a 1.5 Tesla Philips (Acheiva) MRI machine. Inclusion criteria: a. Age group: Any age. b. Both sexes are included. c. Cholesteatoma suspected clinically or by CT examination or known patients with cholesteatoma with suspected post-operative recurrence and prepared for second look surgery. d. Patients underwent primary canal wall up mastoidectomy and were scheduled for second look surgery after one year. Exclusion criteria: a. Patients with contraindication to MR imaging (e.g. pacemaker, metallic implant, severe claustrophobia). Method: MRI will be obtained using 1.5 tesla MRI machines. Diffusion weighted protocol will be operated. The patients were subjected to: consent taking, detailed history taking, clinical general and full otorhinolaryngologic examination, otologic microscopic and endoscopic examination, basic audiologic evaluation, previous $\mathrm{CT}$ if available will 
be obtained for comparison, checking for contraindication to MRI imaging, cleaning of the external ear from wax and other secretions to avoid false positive results, patients will lie supine, patients are asked to breathe quietly and not to move for the duration of the scan. Middle ear and mastoid cavity exploration under surgical microscope, examining all compartments, common sites for residual or recurrent pathology and suspected inflammatory tissues ensuring proper drainage and aeration by using various approaches.

Statistical analysis: Data were tabulated and analyzed using SPSS 19 statistical analysis package software. Statistical analysis will be performed using Microsoft ${ }^{\circledR}$ Excel ${ }^{\circledR}$ version 2010 and Statistical Package for Social Sciences (SPSS ${ }^{\circledR}$ ) for Windows ${ }^{\circledR}$ version 15.0. P value less than 0.05 was considered statistically significant.

\section{RESULTS}

Table (1): Distribution of gender and age of studied patients

\begin{tabular}{|l|c|c|c|}
\hline & No. & $\%$ & \\
\hline Gender & & & \\
Male & 20 & $\mathbf{6 6 . 7}$ & \\
Female & 10 & 33.3 & \\
\hline Age & & & \\
$\quad<18$ years & 4 & $\mathbf{1 3 . 3}$ & \\
$\quad>18$ years & 26 & 86.7 & \\
\hline & Mean & SD & Range \\
\hline Age & 26.9 & 9.4 & $11-56$ \\
\hline Median age (years) & 26 & & \\
\hline
\end{tabular}

This table shows that $66.7 \%$ of the patients were males. The mean age of the patients was 26.9 years \pm 9.4 while the median age was 26 years $13.3 \%$ of the patients were children $<=18$ years of age while $86.7 \%$ were adult patients.

Table (2): Distribution of the side of the lesion

\begin{tabular}{|l|c|c|}
\hline & No. & \% \\
\hline Right side & 19 & $\mathbf{6 3 . 3}$ \\
\hline Left side & 9 & 30.0 \\
\hline Bilateral & 2 & 6.7 \\
\hline
\end{tabular}

This table shows that $63.3 \%$ of the patients had their lesion on the right side while $30.0 \%$ had their lesion on the left side and $6.7 \%$ had bilateral affection.
Table (3): Distribution of positive cholesteatoma by surgical exploration and the MRI finding

\begin{tabular}{|l|c|c|}
\hline & No. & \% \\
\hline Intraoperative & & \\
Negative & 7 & 23.3 \\
Positive & 23 & 76.7 \\
\hline DWI finding & & \\
Negative & 11 & 36.7 \\
Positive & 19 & 63.3 \\
\hline
\end{tabular}

This table shows that only $63.3 \%$ of the patients had positive finding by DWI while $76.7 \%$ of the patients proved positive by intra operative findings.

Table (4): Sensitivity and specificity of DWI in detection of cholesteatoma

\begin{tabular}{|l|c|c|}
\hline & $\begin{array}{c}\text { Negative } \\
\mathbf{N}=\mathbf{7}\end{array}$ & $\begin{array}{c}\text { Positive } \\
\mathbf{N}=\mathbf{2 3}\end{array}$ \\
\hline DWI Negative $\mathrm{N}=11$ & $7(\mathbf{1 0 0 . 0} \%)$ & $4(17.4 \%)$ \\
\hline DWI positive $\mathrm{N}=19$ & $0(0.0 \%)$ & $\mathbf{1 9}(\mathbf{8 2 . 6 \%})$ \\
\hline
\end{tabular}

On comparison of the data produced by MS-EPI DWIs to those produced by intra-operative data/histopathology or follow up, the MS-EPI DWI shows the following diagnostic value according to our study in the detection of primary or recurrent cholesteatoma.

\section{DISCUSSION}

Middle ear cholesteatomas are ectopic keratinized epithelial tissue that grows within the middle ear cavity and mastoid cavity. It has erosive potential affecting the ossicles and the middle ear walls. Due to its potentially serious intracranial and labyrinthine complications, surgery is the treatment of choice with the purpose of eradicating the disease while simultaneously attempting to preserve anatomy and function ${ }^{(\mathbf{1 0})}$. The diagnosis of cholesteatoma is based on clinical findings preferably by otomicroscopy, however it may be obscured by a polyp or granulation tissues and complex anatomical structures ${ }^{(4)}$. The aim of modern otologic surgery for chronic otitis media is to make the ear safe and to preserve or restore the hearing although the former takes the priority over the later. Both (CWUP) and (CWDP) are done depending upon extent of disease, priority of the patient and surgical expertise available. Persistent discharge and recurrent infection after mastoid surgery hallmarks failure at achieving these results. Disease persistence after surgery should be 
carefully considered in the spectrum of complications and development of surgical approach that is both flexible and complete will maximize success in revision surgery. The main objective in revision surgery is eradication of diseased cavities aiming at a well healed dry cavity. Poor execution of proper surgical technique is the important factor contributing to failure of primary surgery this includes high facial ridge, stenotic meatus, badly shaped cavity, bony over hang, large mastoid bowl and residual or recurrent cholesteatoma ${ }^{(11)}$. These anatomical defects act as mechanical barrier preventing self cleaning of cavity and promote disease process by accumulation of debris. The lowering of facial ridge is necessary to prevent this effect by transforming the classical "bean-shaped" cavity into "Round-shaped" cavity. Although intact canal wall procedures have reduced the difficulties associated with open cavities they are more prone to failure. Revision surgery of a chronic ear disease needs extra care because of the possibility of dehiscent or exposed facial nerve, fistula in labyrinth or dural exposure. This may be due to the disease process itself or result of the previous surgery. Familiarization with temporal bone anatomy is a key to the outcome and frequent temporal bone dissection plays an important role in such cases. Preoperative radiological studies are accepted as a standard in many otologic procedures for better understanding of anatomy or pathological changes. Post-contrast T1-weighted MRI imaging has been advocated as a technique for distinguishing granulation tissue from residual cholesteatoma. Cholesteatomas are non-vascular and show no post-contrast enhancement, whereas granulation tissue is poorly vascularized and enhances on delayed images. This technique was able to detect larger cholesteatomas but often missed small residual lesions. Authors have typically advocated post-contrast imaging delays of 30-45 minutes which is inconvenient for patients and reduces practice efficiency, in addition to possible adverse effects of contrast infusion, questioning it's cost benefit efficiency ${ }^{(12)}$. During the recent years, data have been published advocating DWI for evaluation of residual/recurrent cholesteatoma following mastoidectomy or differentiating primary cholesteatoma from other similarly looking pathologies. The DWI technique adds a preparation period before the image acquisition that enhances MR signal intensity attenuation in response to diffusion and other spin motion occurring during this period. Although not fully understood, cholesteatomas are hyperintense on DWI images, like epidermoid cysts, which are histologically identical. This may be due to a combination of T2 and diffusion effects ${ }^{(\mathbf{1 3})}$. When assessing DWI in the evaluation of cholesteatoma, investigators have used a wide range of techniques from traditional spin-echo EPI-based to TSE-based techniques such as HASTE and BLADE (Siemens, Erlangen, Germany). These techniques use a similar method for encoding diffusion, but are different in the method of image acquisition. This methodology significantly impacts the sensitivity to factors such as bulk or physiologic motion and field inhomogeneities ${ }^{(9)}$. The current study was done to highlight the diagnostic value of Multi-shot Echoplanar DWI in the detection of middle ear cholesteatoma. The results were compared to intraoperative data during mastoid surgeries and/ or follow up data. We used a 1.5 Tesla Philips (Acheiva) MRI machine in the radio diagnosis department. We tested 30 patients of all age groups and both sexes with recurrent Cholesteatoma suspected clinically or by CT examination or known patients with cholesteatoma with suspected post-operative recurrence. MR images of the skull base were acquired by using a 1.5-T system with the manufacturer's head coils. The images were obtained in axial and coronal planes using thin cut multi-shot echo-planar diffusion-weighted images with $b$ factors of 0 and $1000 \mathrm{~s} / \mathrm{mm} 2$. ADC maps were also being obtained. Additional sequences were added such as $\mathrm{T} 1$ and $\mathrm{T} 2$ weighted images. Total imaging time was about $15-20$ minutes. MRI images were reviewed by radiologists in radiodiagnosis department. Cholesteatoma was diagnosed by the presence of high signal intensity on T2-weighted sequences, showing high signal intensity and diffusion restriction on diffusionweighted imaging without calculation of the ADC value yet the ADC maps were used to exclude the presence of T2 shine through effects. From the 30 cases we examined, 19 cases $(63 \%)$ showed areas of DWI restriction within the middle ear denoting primary or recurrent cholesteatoma. The smallest size of cholesteatoma detected was $4.5 \mathrm{~mm}$. All 19 cases that showed evidence of recurrent cholesteatoma on MS-EPI DWI MRI images 
underwent second look mastoid surgery will all cases $(100 \%)$ showing intra-operative evidence of cholesteatoma. Of the 11 cases that were negative for cholesteatoma on DWIs, 9 of these cases underwent second look mastoid surgery with 4 cases (44 \%) showing small cholesteatomas measuring less than $4 \mathrm{~mm}$ (that were not visualized by DWIs) while the other 5 cases $(56 \%)$ showed only granulation and/or inflammatory tissue with no evidence of cholesteatoma. The other 2 cases that didn't undergo surgery after MRI imaging were treated medically with significant improvement of their symptoms. Specificity of MS-EPI in detection of cholesteatoma according to our study was $100 \%$ which correlates well with multiple previous studies using both EPI and NonEPI techniques. The least size of cholesteatoma detected by our study was $4.5 \mathrm{~mm}$ which is slightly better than previous studies using SS-EPI such as Vercruysse et al. ${ }^{(14)}(5 \mathrm{~mm})$, Jeunen et al. ${ }^{(15)}(5$ $\mathrm{mm})$ and Aikele et al. ${ }^{(16)}(5 \mathrm{~mm})$ but less than other studies using Non-EPI DWI such as De Foer et $\boldsymbol{a l} .{ }^{(8)}(2 \mathrm{~mm})$. Our study revealed that the use of thin cut MS-EPI is a beneficial tool in the evaluation of both primary and recurrent cholesteatoma with excellent specificity that reduces the need of second look surgeries in multiple cases. It also revealed improved sensitivity and smallest detectable size compared to SS-EPI techniques. However, MS-EPI DWIs show relatively less sensitivity compared to non-EPI techniques with the smallest detectable size being about $4.5 \mathrm{~mm}$. Authors such as Migirov et al. (6) stated that leaving such small cholesteatomas is considered safe, and some authors propose followup studies to detect these lesions once they have grown larger. The necessity for such follow-up studies and the interval at which they should be performed, are issues still to be determined. In addition, Non-EPI DWIs is unavailable in many MR imaging systems including our system, while MS-EPI requires no special installation and is, therefore, widely available.

\section{CONCLUSION}

DWI is a beneficial tool in the evaluation of recurrent cholesteatoma with excellent specificity that reduces the need of second look surgeries in multiple cases. The use of multi-shot diffusion weighted techniques improved its sensitivity significantly compared to single shot techniques. It still could not detect lesions smaller than $4 \mathrm{~mm}$.

\section{CONFLICTS OF INTEREST}

There are no conflicts of interest.

\section{REFERENCES}

1. Swartz JD (1996): Temporal bone inflammatory disease. Head and Neck Imaging, 3rd ed. St. Louis: Mosby.

2. Semaan MT and Megerian CA (2006): The pathophysiology of cholesteatoma. Otolaryngol Clin North Am., 39:114359.

3. Gotz A, Goldenberg D, Netzer A et al. (1999): Cholesteatomas associated with ventilation tube insertion. Arch Otolaryngol Head Neck Surg., 125(7):754-7.

4. Corrales C and Blevins NH (2013): Curr Opin Otolaryngol Head NeckSurg., 21(5):461-7.

5. Li PM, Linos E, Gurgel RK, Fischbein NJ, Blevins NH (2013): Evaluating the utility of non-echo-planar diffusionweighted imaging in the preoperative evaluation of cholesteatoma: a metaanalysis. Laryngoscope, 123(5):1247-50.

6. Migirov L, Tal S, Eyal A, Kronenberg J (2009): MRI, not CT, to rule out recurrent cholesteatoma and avoid unnecessary second-look mastoidectomy. Isr Med Assoc J., 11(3): 144-146.

7. Schwab SA, Eberle S, Adamietz B, Kuefner MA, Kramer M, Uder M, Lell M (2012): Comparison of 128-section single-shot technique with conventional spiral multisection CT for imaging of the temporal bone. AJNR Am J Neuroradiol., 33(4):E55-60.

8. De Foer B, Vercruysse JP, Offeciers E, Casselman E (2008): MR of cholesteatoma.Recent advantages in Otolaryngology, The Royal society of Medicine Press, London. 
9. Schwartz KM, Lane JI, Bolster BD, Neff BA (2011): The Utility of DiffusionWeighted Imaging for Cholesteatoma Evaluation. AJNR Am J Neuroradiol., 32:430 -36.

10. Probst $R$ (2006): The middle ear. In: Probst R, Grevers G, Iro H, eds. Basic otorhinolaryngology: a step-by-step learning guide.2nd ed. Stuttgart, Germany: Georg Thieme Verlag., 227253.

11. Shirazi MA, Muzaffar K, Leonetti JP and Marzo S (2006): Surgical Treatment of Pediatric Cholesteatomas. The Laryngoscope, 116:1603-1607.

12. Williams MT, Ayache D, Alberti $C$ et al. (2003): Detection of postoperative residual cholesteatoma with delayed contrast enhanced MR imaging. Eur Radiol., 13: 169-74.

13. Schaefer PW, Grant PE, Gonzalez RG (2000): Diffusion- weighted MR imaging of the brain. Radiology, 217: 331-45
14. Vercruysse JP, De Foer B, Pouillon M, Somers T, Casselman J, Offeciers $\mathbf{E}$ (2006): The value of diffusion-weighted MR imaging in the diagnosis of primary acquired and residual cholesteatoma: a surgical verified study of 100 patients. Eur Radiol., 16:1461- 1467.

15. Jeunen G, Desloovere $C$, Hermans R, Vandecaveye $V$ (2008): The value of magnetic resonance imaging in the diagnosis of residual or recurrent acquired cholesteatoma after canal wall up tympanoplasty. Otol Neurotol., 28:1618. 14.

16. Aikele P, Kittner T, Offergeld C, Kaftan H, Hüttenbrink KB, Laniado M (2003): Diffusion-weighted MR imaging of cholesteatoma in pediatric and adult patients who have undergone middle ear surgery. AJR Am J Roentgenol., 181:261265. 